\title{
Extension of Generalized Bernoulli Learning Models
}

\author{
B. S. El-Desouky, F. A. Shiha, A. M. Magar \\ Department of Mathematics, Faculty of Science, Mansoura University, Mansoura, Egypt \\ Email: bdesouky@yahoo.com, fshiha@yahoo.com, alia.ma16@yahoo.com
}

Received 31 December 2014; accepted 13 January 2015; published 14 January 2015

Academic Editor: Antonio Hervás Jorge, Department of Applied Mathematics, Universidad Politécnica de Valencia, Spain

Copyright (C) 2015 by authors and Scientific Research Publishing Inc.

This work is licensed under the Creative Commons Attribution International License (CC BY).

http://creativecommons.org/licenses/by/4.0/

(c) (i) Open Access

\section{Abstract}

In this article, we study the generalized Bernoulli learning model based on the probability of success $p_{i}=\alpha_{i} / n$ where $i=1,2, \cdots, n \quad 0<\alpha_{1}<\alpha_{2}<\cdots<\alpha_{n} \leq n$ and $n$ is positive integer. This gives the previous results given by Abdulnasser and Khidr [1], Rashad [2] and EL-Desouky and Mahfouz [3] as special cases, where $p_{i}=i / n \quad p_{i}=i^{2} / n^{2}$ and $p_{i}=i^{p} / n^{p}$ respectively. The probability function $P\left(W_{n}=k\right)$ of this model is derived, some properties of the model are obtained and the limiting distribution of the model is given.

\section{Keywords}

Stirling Numbers, Bernoulli Learning Models, Comtet Numbers, Inclusion-Exclusion Principle

\section{Introduction}

In industry, training programmes are conducted with the aim of training new workers to do particular job repeatedly every day. It is assumed that a particular trainee will show progress proportional to the number of days he attends the program, otherwise his ability will be different from one day to another, see [1] [4].

Let $n$ be the length of a programme in days and $l$ the number of repetitions of the job per day a trainee has to do. If a trainee is responding to the instructions, it would be reasonable to assume the probability that he will do a single job right, i.e. the probability of success on the $i^{\text {th }}$ day is $p_{i}=i / n$, see Abdulnasser and Khidr [1], and hence the probability that he will do $x$ jobs correctly out of $l$ jobs on the $i^{\text {th }}$ day is $\left(\begin{array}{l}l \\ x\end{array}\right)(i / n)^{x}$ 
$(1-i / n)^{1-x}, \quad x=0,1, \cdots, l$ and $i=0,1, \cdots, n$.

When a trainee is not responding to the instructions, $p_{i}$ will be a constant $p, 0<p<1$. To test whether a trainee is responding or not, we test if $p_{i}$ is varying or sustaining a constant value $p$. This can be done by computing the total number of jobs that have been done correctly over the whole period of the program.

Let $X_{n, i}^{l}$ stand for the number of jobs done correctly out of $l$ jobs on $i^{\text {th }}$ day, $i=1,2, \cdots, n, \quad l=1,2, \cdots$ and $W_{n}^{l}=\sum_{i=1}^{n} X_{n, i}^{l}, \quad l \leq W_{n}^{l} \leq n l$. In case $p_{i}=p, 0<p<1$, the distribution of $W_{n}^{l}$ will be $B(n l, p)$.

In this article, we study a generalization of Bernoulli learning model based on probability of success $p_{i}=\alpha_{i} / n$ where $n$ positive integer, $\alpha_{i}$ are real numbers, $i=1,2, \cdots, n$, and $0<\alpha_{1}<\alpha_{2}<\cdots<\alpha_{n} \leq n$ and $n$ is positive integer. This gives the previous results given in [1]-[3] as special cases, where $p_{i}=i / n$ $p_{i}=i^{2} / n^{2}$ and $p_{i}=i^{p} / n^{p}$ respectively. In Section 2, the probability function $P\left(W_{n}=k\right)$ of this model and some properties of the model are obtained. In Section 3, we derive the limiting distribution of the model. Finally, in Section 4, we discuss some special cases.

\section{The Generalized Bernoulli Learning Model}

Theorem 1. The distribution function of $W_{n}$ is

$$
P\left(W_{n}=k\right)=(-1)^{k} \sum_{m=k}^{n} \frac{s_{\bar{\alpha}}(n+1, n+1-m)}{m^{n}}\left(\begin{array}{l}
m \\
k
\end{array}\right),
$$

where $W_{n}=\sum_{i=1}^{n} X_{n, i}^{1}, \quad X_{n, i}^{1} \approx B\left(1, \alpha_{i} / n\right)$.

Proof. To derive the distribution of Bernoulli learning model based on the sum of the independent random variable $\left\{X_{n, i}^{1}\right\}_{i=1}^{n}, \quad i=1,2, \cdots, n$,

where the probability of success is $p_{i}=\alpha_{i} / n$ we define the event $E_{i}$ as the event $X_{n, i}^{1}, i=1,2, \cdots, n$ see [5], and the sum

$$
\begin{aligned}
P(n, k) & =\sum_{1 \leq i_{1}<\cdots<i_{k} \leq n} P\left(E_{i_{1}}, E_{i_{2}}, \cdots, E_{i_{k}}\right) \\
& =\sum_{1 \leq i_{1}<\cdots<i_{k} \leq n} P\left(X_{n, i_{1}}^{1}=1, X_{n, i_{2}}^{1}=1, \cdots, X_{n, i_{k}}^{1}=1\right) \\
& =\sum_{1 \leq i_{1}<\cdots<i_{k} \leq n} \frac{\alpha_{i_{1}}}{n} \frac{\alpha_{i_{2}}}{n} \cdots \frac{\alpha_{i_{k}}}{n}=\frac{1}{n^{k}} \sum_{1 \leq i_{1}<\cdots<i_{k} \leq n} \alpha_{i_{1}} \alpha_{i_{2}} \cdots \alpha_{i_{k}} \\
& =(-1)^{k} s_{\bar{\alpha}}(n+1, n+1-k) / n^{k},
\end{aligned}
$$

where $s_{\bar{\alpha}}(n, k)$ the generalized Stirling number of the first kind (Comtet numbers), defined by Comtet in [6] [7] as follows

$$
\left(x-\alpha_{0}\right)\left(x-\alpha_{1}\right) \cdots\left(x-\alpha_{n-1}\right)=\sum_{k=0}^{n} s_{\bar{\alpha}}(n, k) x^{k}
$$

where $\bar{\alpha}=\left(\alpha_{0}, \alpha_{1}, \cdots, \alpha_{n-1}\right)$, for more details, see [8] and [9].

Employing the inclusion-exclusion principle, see [5], we get

$$
\begin{aligned}
P\left(W_{n} \geq k\right) & =\sum_{m=k}^{n}(-1)^{m-k}\left(\begin{array}{c}
m-1 \\
k-1
\end{array}\right) P(n, m) \\
& =\sum_{m=k}^{n}(-1)^{m-k}\left(\begin{array}{c}
m-1 \\
k-1
\end{array}\right) \frac{(-1)^{m} s_{\bar{\alpha}}(n+1, n+1-m)}{n^{m}} \\
& =(-1)^{k} \sum_{m=k}^{n} \frac{s_{\bar{\alpha}}(n+1, n+1-m)}{n^{m}}\left(\begin{array}{c}
m-1 \\
k-1
\end{array}\right),
\end{aligned}
$$

then 


$$
P\left(W_{n} \geq k+1\right)=(-1)^{k+1} \sum_{m=k+1}^{n} \frac{s_{\bar{\alpha}}(n+1, n+1-m)}{n^{m}}\left(\begin{array}{c}
m-1 \\
k
\end{array}\right),
$$

hence

$$
\begin{gathered}
P\left(W_{n}=k\right)=P\left(W_{n} \geq k\right)-P\left(W_{n} \geq k+1\right) \\
P\left(W_{n}=k\right)=(-1)^{k} \sum_{m=k}^{n} \frac{s_{\bar{\alpha}}(n+1, n+1-m)}{n^{m}}\left(\begin{array}{c}
m-1 \\
k-1
\end{array}\right)-(-1)^{k+1} \sum_{m=k+1}^{n} \frac{s_{\bar{\alpha}}(n+1, n+1-m)}{n^{m}}\left(\begin{array}{c}
m-1 \\
k
\end{array}\right) \\
=(-1)^{k}\left(\begin{array}{c}
k-1 \\
k-1
\end{array}\right) \frac{s_{\bar{\alpha}}(n+1, n+1-k)}{n^{k}}+(-1)^{k} \sum_{m=k+1}^{n} \frac{s_{\bar{\alpha}}(n+1, n+1-m)}{n^{m}}\left(\begin{array}{c}
m-1 \\
k-1
\end{array}\right) \\
+(-1)^{k} \sum_{m=k+1}^{n} \frac{s_{\bar{\alpha}}(n+1, n+1-m)}{n^{m}}\left(\begin{array}{c}
m-1 \\
k
\end{array}\right) \\
=(-1)^{k} \frac{s_{\bar{\alpha}}(n+1, n+1-k)}{n^{k}}+(-1)^{k} \sum_{m=k+1}^{n} \frac{s_{\bar{\alpha}}(n+1, n+1-m)}{n^{m}}\left\{\left(\begin{array}{c}
m-1 \\
k-1
\end{array}\right)+\left(\begin{array}{c}
m-1 \\
k
\end{array}\right)\right\} \\
=(-1)^{k} \frac{s_{\bar{\alpha}}(n+1, n+1-k)}{n^{k}}+(-1)^{k} \sum_{m=k+1}^{n} \frac{s_{\bar{\alpha}}(n+1, n+1-m)}{n^{m}}\left(\begin{array}{l}
m \\
k
\end{array}\right),
\end{gathered}
$$

this yields (1).

Lemma 1.

$$
\begin{gathered}
\mu_{W_{n}}=E\left(W_{n}\right)=\sum_{i=1}^{n} \frac{\alpha_{i}}{n}, \\
\operatorname{Var}\left(W_{n}\right)=\frac{1}{n^{2}}\left(2 \sum_{1 \leq i_{1} \leq i_{2} \leq n} \alpha_{i_{1}} \alpha_{i_{2}}+n \sum_{i=1}^{n} \alpha_{i}-\left(\sum_{i=1}^{n} \alpha_{i}\right)^{2}\right) .
\end{gathered}
$$

Proof. Consider the pair of inverse relation, see [10]

$$
a_{k}=\sum_{m=k}\left(\begin{array}{l}
m \\
k
\end{array}\right) b_{m}, \quad b_{k}=\sum_{m=k}(-1)^{m+k}\left(\begin{array}{l}
m \\
k
\end{array}\right) a_{m} .
$$

Then using (1), let

$$
g_{k}=P\left(W_{n}=k\right)=(-1)^{k} \sum_{m=k}\left(\begin{array}{l}
m \\
k
\end{array}\right) \frac{s_{\bar{\alpha}}(n+1, n+1-k)}{n^{m}} .
$$

Hence from (4), we get

$$
s_{\bar{\alpha}}(n+1, n+1-k) n^{-k}=(-1)^{k} \sum_{m=k}\left(\begin{array}{l}
m \\
k
\end{array}\right) g_{m},
$$

and setting $k=1$, we have

$$
s_{\bar{\alpha}}(n+1, n) n^{-1}=-\sum_{m=k}^{n} m g_{m}=-E\left(W_{n}\right) .
$$

But we have, see [7]

$$
s_{\bar{\alpha}}(n, k)=(-1)^{n-k} \sum_{1 \leq i_{1}<i_{2}<\cdots<i_{n-k} \leq n} \alpha_{i_{1}} \alpha_{i_{2}} \cdots \alpha_{i_{n-k}} .
$$

Thus $s_{\bar{\alpha}}(n+1, n)=-\sum_{i=1}^{n} \alpha_{i}$ and this yields (2).

If putting $k=2$ in (5), we get

$$
s_{\bar{\alpha}}(n+1, n-1) n^{-2}=\sum_{m=2}^{n}\left(\begin{array}{l}
m \\
2
\end{array}\right) g_{m}=\sum_{m=2}^{n} \frac{m(m-1)}{2} g_{m}=\frac{1}{2} \sum_{m=2}^{n} m^{2} g_{m}-\frac{1}{2} \sum_{m=2}^{n} m g_{m}=\frac{1}{2} E\left(\left(W_{n}\right)^{2}\right)-\frac{1}{2} E\left(W_{n}\right),
$$


using (7), we have $s_{\bar{\alpha}}(n+1, n-1)=\sum_{1 \leq i_{1}<i_{2} \leq n} \alpha_{i_{1}} \alpha_{i_{2}}$, then

$$
E\left(\left(W_{n}\right)^{2}\right)=2 n^{-2} \sum_{1 \leq i_{1}<i_{2} \leq n} \alpha_{i_{1}} \alpha_{i_{2}}+\sum_{i=1}^{n} \frac{\alpha_{i}}{n}
$$

hence

$$
\begin{gathered}
\operatorname{Var}\left(W_{n}\right)=E\left(\left(W_{n}\right)^{2}\right)-\left(E\left(W_{n}\right)\right)^{2} \\
\operatorname{Var}\left(W_{n}\right)=2 n^{-2} \sum_{1 \leq i_{1}<i_{2} \leq n} \alpha_{i_{1}} \alpha_{i_{2}}+\sum_{i=1}^{n} \frac{\alpha_{i}}{n}-\left(\sum_{i=1}^{n} \frac{\alpha_{i}}{n}\right)^{2},
\end{gathered}
$$

this yields (3).

\section{Limiting Distribution of the Bernoulli Learning Model}

In this section we study the limiting distribution of the Bernoulli learning model based on the probability with success $\alpha_{i} / n$.

Theorem 2. Let $W_{n}=\sum_{i=1}^{n} X_{n, i}^{1}$ where $X_{n, i}^{1} \approx B\left(1, \alpha_{i} / n\right)$ and $X_{n, i}^{1}$ are independent random variables. Then $\lim _{n \rightarrow \infty} M_{Z_{n}}=\exp \left(t^{2} / 2\right)$ where $Z_{n}=\frac{W_{n}-\mu_{W_{n}}}{\sigma_{W_{n}}}$ i.e. $Z_{n}$ is $N(0,1)$ as $n \rightarrow \infty$.

Proof. The moment generating function of $Z_{n}$ is

$$
M_{Z_{n}}(t)=M_{\frac{W_{n}-\mu_{W_{n}}}{\sigma_{W_{n}}}}=\mathrm{e}^{\left(-\frac{\mu_{W_{n}}}{\sigma_{W_{n}}}\right) t} M_{W_{n}}\left(t / \sigma_{W_{n}}\right),
$$

and the moment generating function of $W_{n}$ is

$$
\begin{gathered}
M_{W_{n}}\left(t / \sigma_{W_{n}}\right)=E\left(\mathrm{e}^{W_{n}\left(t / \sigma_{W_{n}}\right)}\right), \quad W_{n}=\sum_{i=1}^{n} X_{n, i}^{1} \text {, hence } \\
M_{W_{n}}\left(t / \sigma_{W_{n}}\right)=\prod_{i=1}^{n} E\left(\mathrm{e}^{X_{n, i}^{1}\left(t / \sigma_{W_{n}}\right)}\right)=\prod_{i=1}^{n}\left(\sum_{x=0}^{1} \mathrm{e}^{x t / \sigma_{W_{n}}}\left(\begin{array}{l}
1 \\
x
\end{array}\right)\left(\frac{\alpha_{i}}{n}\right)^{x}\left(1-\frac{\alpha_{i}}{n}\right)^{1-x}\right)=\prod_{i=1}^{n}\left(\frac{n-\alpha_{i}}{n}+\frac{\alpha_{i}}{n} \mathrm{e}^{t / \sigma_{W_{n}}}\right), \quad \text { then } \\
M_{Z_{n}}(t)=\mathrm{e}^{\left(-\frac{\mu_{W_{n}}}{\sigma_{W_{n}}}\right) t} \prod_{i=1}^{n}\left(1+\frac{\alpha_{i}}{n}\left(\mathrm{e}^{t / \sigma w_{n}}-1\right)\right),
\end{gathered}
$$

therefore, we have

$$
\begin{aligned}
\ln M_{Z_{n}}(t) & =\frac{-\mu_{W_{n}} t}{\sigma_{W_{n}}}+\sum_{i=1}^{n} \ln \left(1+\frac{\alpha_{i}}{n}\left(\mathrm{e}^{t / \sigma_{W_{n}}}-1\right)\right)=\frac{-\mu_{W_{n}} t}{\sigma_{W_{n}}}+\sum_{i=1}^{n} \sum_{k=1}^{\infty} \frac{(-1)^{k-1}}{k}\left(\frac{\alpha_{i}}{n}\left(\mathrm{e}^{t} / \sigma_{W_{n}}-1\right)\right)^{k} \\
& =\frac{-\mu_{W_{n}} t}{\sigma_{W_{n}}}+\sum_{i=1}^{n} \sum_{k=1}^{\infty} \frac{(-1)^{k-1}}{k}\left(\frac{\alpha_{i}}{n}\right)^{k}\left(\sum_{j=1}^{\infty} \frac{\left(t / \sigma_{W_{n}}\right)^{j}}{j !}\right)^{k} \\
& =\frac{-\mu_{W_{n}} t}{\sigma_{W_{n}}}+\sum_{i=1}^{n} \frac{\alpha_{i}}{n}\left(\frac{t}{\sigma_{W_{n}}}+\frac{1}{2 !} \frac{t^{2}}{\sigma_{W_{n}}^{2}}+\frac{1}{3 !} \frac{t^{3}}{\sigma_{W_{n}}^{3}}+\cdots\right)-\sum_{i=1}^{n} \frac{\alpha_{i}^{2}}{2 n^{2}}\left(\frac{t}{\sigma_{W_{n}}}+\frac{1}{2 !} \frac{t^{2}}{\sigma_{W_{n}}^{2}}+\frac{1}{3 !} \frac{t^{3}}{\sigma_{W_{n}}^{3}}+\cdots\right)^{2}+\cdots \\
& =\frac{-\mu_{W_{n}} t}{\sigma_{W_{n}}}+\frac{t}{\sigma_{W_{n}}} \sum_{i=1}^{n} \frac{\alpha_{i}}{n}+\frac{1}{2 !} \frac{t^{2}}{\sigma_{W_{n}}^{2}} \sum_{i=1}^{n}\left(\frac{\alpha_{i}}{n}-\frac{\alpha_{i}^{2}}{n^{2}}\right)+O(1 / n),
\end{aligned}
$$


by using (2) and (3), we obtain

$$
\begin{gathered}
\ln M_{Z_{n}}(t)=\frac{t^{2}}{2}+O(1 / n), \quad \text { hence } \\
\lim _{n \rightarrow \infty} M_{Z_{n}}(t) \cong \exp \left(t^{2} / 2\right)
\end{gathered}
$$

which is the moment generating function of standard normal distribution $N(0,1)$.

\section{Some Special Cases}

In this section we discuss some special cases as follows.

i) Setting the probability of successes $p_{i}=\frac{i}{n}$ we have the results derived in [1], as special case

Theorem 3. The distribution of $W_{n}^{1}$ is given by [1]

$$
P\left(W_{n}^{1}=k\right)=(-1)^{k} \sum_{m=k}^{n} \frac{s(n+1, n+1-m)}{n^{m}}\left(\begin{array}{c}
m \\
k
\end{array}\right), \quad k=1,2, \cdots, n,
$$

where $s(n, k)$ are the usual stirling numbers of the first kind, see [10].

Also, they obtained the limiting distribution of learning model, mean and variance as follows.

Theorem 4. Let $W_{n}^{1}=\sum_{i=1}^{n} X_{i}$, where $X_{i} \approx B(1, i / n)$ and $X$ 's are independent random variables. Then $\lim _{n \rightarrow \infty} M_{Z_{n}}=\mathrm{e}^{t^{2} / 2}$ where $Z_{n}=\frac{W_{n}-\mu_{W_{n}}}{\sigma_{W_{n}}}$ i.e. $Z_{n}$ has $N(0,1)$ as $n \rightarrow \infty$.

Lemma 2.

$$
\mu_{W_{n}^{1}}=\frac{n+1}{2}, \quad \sigma_{W_{n}^{1}}^{2}=\frac{n^{2}-1}{6 n} .
$$

ii) Setting the probability of successes $p_{i}=\left(\frac{i}{n}\right)^{2}$ we have the results derived in [2], as special case

Theorem 5. The distribution of $W_{n}^{1}$ is given by [2]

$$
P\left(W_{n}^{1}=k\right)=(-1)^{n+1+k} \sum_{m=k}^{n}\left(\begin{array}{l}
m \\
k
\end{array}\right)\left(\frac{1}{n^{2 m}}\right)^{2(n+1-k)} \sum_{l=0} s(n+1, l) s(n+1,2(n+1-k)-l) .
$$

Lemma 3.

$$
\mu_{W_{n}}=\frac{(n+1)(2 n+1)}{6 n}, \text { and } \sigma_{W_{n}}^{2}=\frac{4 n^{4}+1}{30 n^{3}}
$$

iii) Setting the probability of successes $p_{i}=\left(\frac{i}{n}\right)^{p}$ we have the results derived in [3], as special case

Theorem 6.

$$
P\left(W_{n}=k\right)=(-1)^{k} \sum_{m=k}^{n}\left(\begin{array}{c}
m \\
k
\end{array}\right) \frac{s_{p}(n+1, n+1-m)}{n^{p m}},
$$

where $W_{n}=\sum_{i=1}^{n} X_{n, i}^{1}, \quad X_{n, i}^{1} \approx B\left(1, i^{p} / n^{p}\right)$ and $s_{p}(n, k), p$-Stirling numbers, see [11] [12].

Theorem 7. Let $W_{n}^{1}=\sum_{i=1}^{n} X_{n, i}^{1}$ where $X_{n, i}^{1} \approx B\left(1, i^{p} / n^{p}\right)$ and $X_{n, i}^{1}$ are independent random variables.

Then $\lim _{n \rightarrow \infty} M_{Z_{n}}=\mathrm{e}^{t^{2} / 2}$ where $Z_{n}=\frac{W_{n}-\mu_{W_{n}}}{\sigma_{W_{n}}}$ i.e. $Z_{n}$ has $N(0,1)$ as $n \rightarrow \infty$. 


\section{Lemma 4.}

$$
\begin{gathered}
\mu_{W_{n}}=E\left(W_{n}\right)=\sum_{i=1}^{n} \frac{i^{p}}{n^{p}}, \quad \text { and } \\
\operatorname{Var}\left(W_{n}\right)=\frac{1}{n^{2 p}}\left(2 \sum_{1 \leq i_{1}<i_{2} \leq n} i_{1}^{p} i_{2}^{p}+n^{p} \sum_{i=1}^{n} i^{p}-\left(\sum_{i=1}^{n} i^{p}\right)^{2}\right) .
\end{gathered}
$$

\section{Conclusion}

Our main goal of this work is concerned with studying the extension of generalized Bernoulli learning model with probability of success $p_{i}=\alpha_{i} / n \quad i=1,2, \cdots, n, \quad 0<\alpha_{1}<\alpha_{2}<\cdots<\alpha_{n} \leq n$ and $n$ is positive integer. Some previous results, see [1]-[3], are concluded as special cases of our result, that is for $p_{i}=i / n \quad p_{i}=i^{2} / n^{2}$ and $p_{i}=i^{p} / n^{p}$ respectively. The mean and variance of the model are obtained. Finally, the limiting distribution of the general model is derived. This model has many applications in industry, specially for training programmes.

\section{References}

[1] Abdulnasser, T. and Khidr, A.M. (1981) On the Distribution of a Sum of Non-Identical Independent Binomials. Journal of University of Kuwait, 8, 109-115.

[2] Rashad, A.M. (1998) On the Sum of Non-Identical Random Variables. M.Sc. Thesis, Faculty of Science, Aswan.

[3] EL-Desouky, B.S. and Mahfouz, K.M. (2012) A Generalization of Bernolli Learning Models. Kuwait Journal of Science and Engineering, 39, 31-44.

[4] Janardan, K.G. (1997) Bernoulli Learing Models: Uppulur numbers. In: Balakrishnan, N., Ed., Advances in Combinatorial Methods and Applications to Probability and Statistics, Birkhäuser. Statistics for Industry and Technology, Boston, 471-480. http://dx.doi.org/10.1007/978-1-4612-4140-9_29

[5] Parzen, E. (1960) Modern Probability Theory and Its Applications. John Wiley \& Sons, Hoboken.

[6] Comtet, M.L. (1972) Nombers de Stirling generoux et functions symetriques. C. R. Acad. Sci. Paris, Ser. A, 275, 747-750.

[7] Comtet, M.L. (1974) Advanced Combinatorics. Reidel, Dordrecht-Holland. http://dx.doi.org/10.1007/978-94-010-2196-8

[8] EL-Desouky, B.S. and Cakić, N.P. (2011) Generalized Higher Order Stirling Numbers. Mathematical and Computer Modelling, 54, 2848-2857. http://dx.doi.org/10.1016/j.mcm.2011.07.005

[9] EL-Desouky, B.S., Cakić, N.P. and Mansour, T. (2010) Modified Approach to Generalized Stirling Numbers via Differential Operators. Applied Mathematics Letters, 23, 115-120. http://dx.doi.org/10.1016/j.aml.2009.08.018

[10] Riordan, J. (1968) Combinatorial Identities. John Wiley \& Sons, New York. London, and Sydney.

[11] Beardon, A.F. (1996) Sums of Powers of Integers. The American Mathematical Monthly, 103, 201-213. http://dx.doi.org/10.1016/j.aml.2009.08.018

[12] Sun, Y. (2006) Two Classes of p-Stirling Numbers. Discrete Mathematics, 306, 2801-2805. http://dx.doi.org/10.1016/j.disc.2006.05.016 
Scientific Research Publishing (SCIRP) is one of the largest Open Access journal publishers. It is currently publishing more than 200 open access, online, peer-reviewed journals covering a wide range of academic disciplines. SCIRP serves the worldwide academic communities and contributes to the progress and application of science with its publication.

Other selected journals from SCIRP are listed as below. Submit your manuscript to us via either submit@scirp.org or Online Submission Portal.
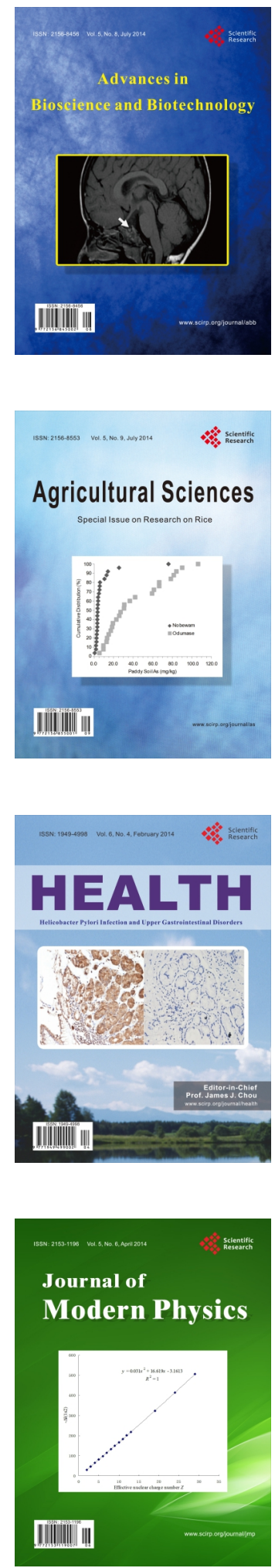
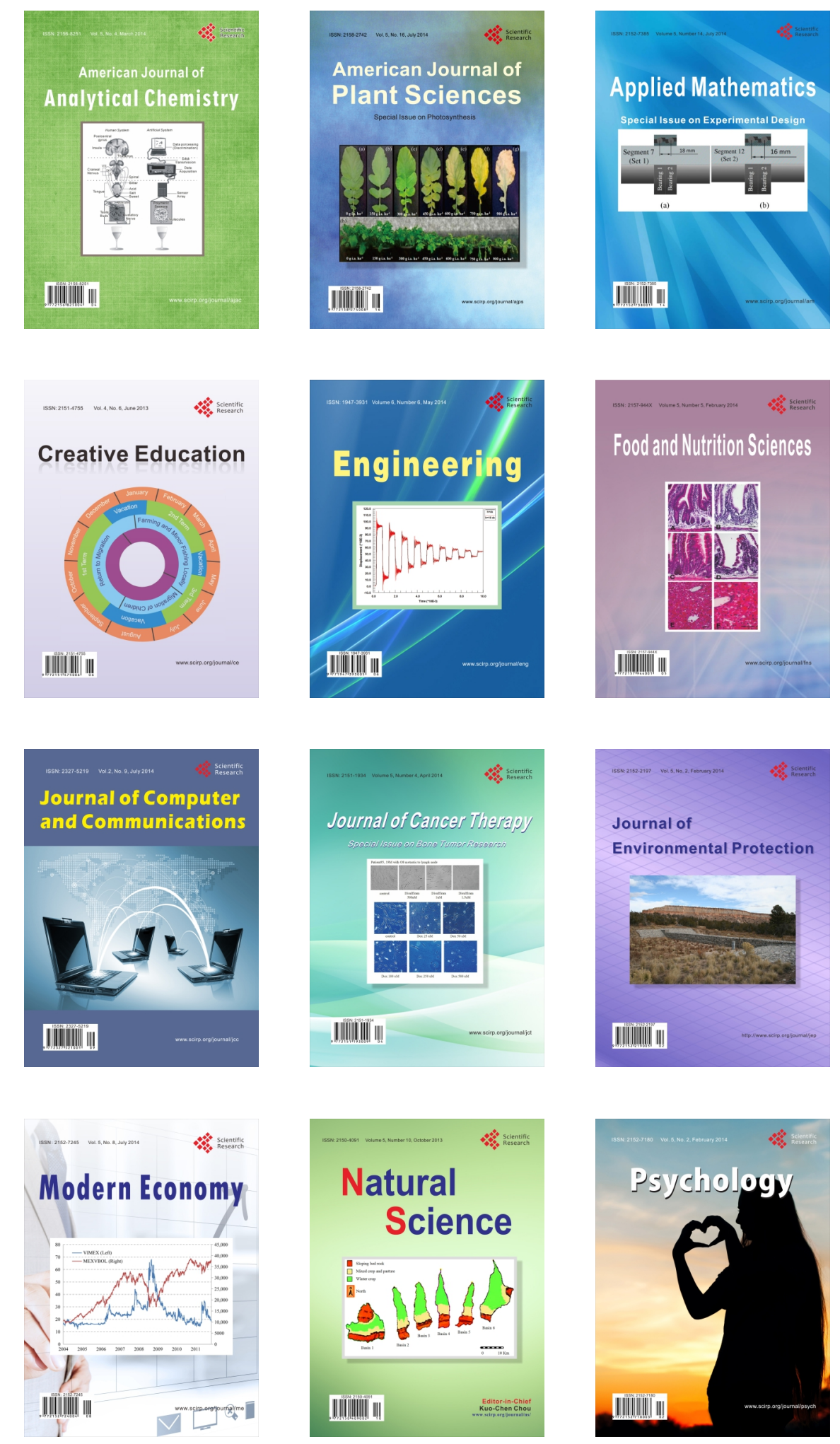